\title{
Capacité des sols forestiers tropicaux de Guyane et de la Réunion à dépolluer les bois imprégnés de biocides
}

\begin{abstract}
Alba ZAREMSKI ${ }^{1}$
LOUIS GASTONGUAY

Clara ZAREMSKI ${ }^{1}$

Fanny ChAFfanel ${ }^{3}$

Gaétan LE FLOCH ${ }^{3}$

Jacques BEAUCHÊNE ${ }^{4}$
\end{abstract}

${ }^{1}$ Cirad

Umr Agap-Gfp

Amélioration génétique et adaptation des plantes méditerranéennes et tropicales TA A-108/01

Avenue Agropolis

34398 Montpellier Cedex 5

France

${ }^{2}$ Institut de recherche d'Hydro-Québec 1800, boulevard Lionel Boulet

Varennes, J3X 1S1

Canada

\section{Université de Brest}

Laboratoire universitaire de biodiversité et écologie microbienne (EA3882)

Technopôle Brest Iroise

29280 Plouzané

France

${ }^{4}$ Cirad

Umr Ecofog

BP 701

97387 Kourou Cedex

France

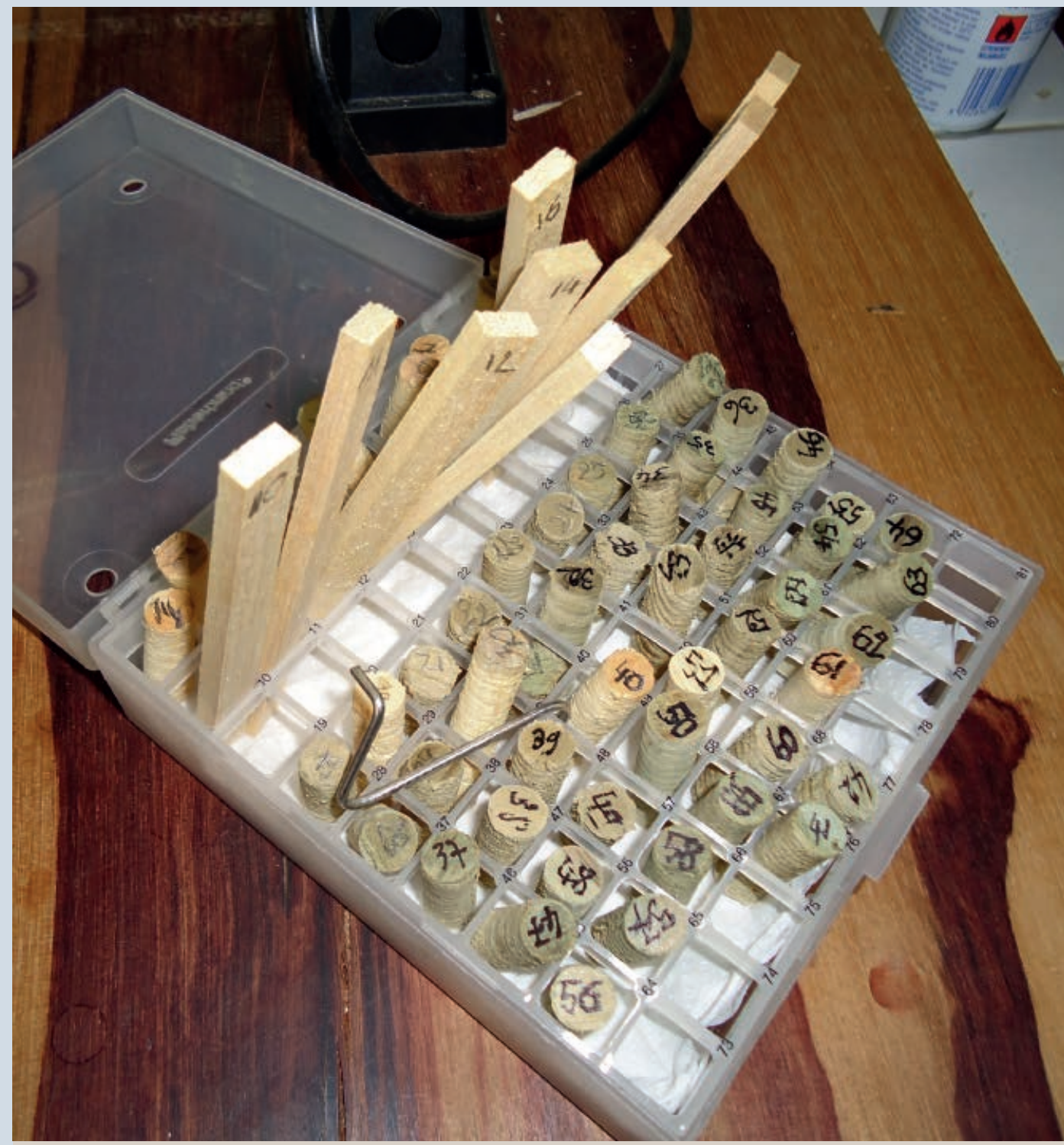

Photo 1.

Éprouvettes traitées et témoins. On différencie les éprouvettes traitées des témoins par leur couleur bleutée.

Photo A. Zaremski. 


\section{RÉSUMÉ}

\section{CAPACITÉ DES SOLS FORESTIERS TROPICAUX DE GUYANE ET DE LA RÉUNION À DÉPOLLUER LES BOIS IMPRÉGNÉS DE BIOCIDES}

Le matériau bois a depuis longtemps fait l'objet de traitements fongicides ou insecticides dont l'impact sur les sols après lessivage constitue un réel problème écologique. À ce jour, l'essentiel des études sur la dégradation de ces produits toxiques a été mené à partir des micro-organismes isolés en laboratoire. La présente étude a cherché à affiner les connaissances concernant ces microorganismes, en particulier les champignons lignivores dégradant les polluants in situ, pour lesquels peu de données sont en fait disponibles. En vue de dépolluer des bois traités, la capacité des micro-organismes lignivores issus de sols forestiers tropicaux de la Guyane et de la Réunion pour dégrader des biocides toxiques a été évaluée. II s'agit du pentachlorophénol (PCP) et des composés à base de cuivre chrome arsenic (CCA). Le suivi de la dégradation d'éprouvettes de pin rouge, Pinus resinosa, montre que le sol de Guyane est plus performant que celui de la Réunion en termes d'activité microbienne vis-à-vis de ces deux biocides. Une différence significative de la perte de masse des éprouvettes de pin rouge traitées au CCA et au PCP peut aller du simple au double (respectivement $18 \%$ et $30 \%)$. Ces résultats confirment que le CCA est moins lessivable et moins dégradable par les microorganismes de ces sols que le PCP. $D$ 'après le barème des pertes de masse en essais de laboratoire, ces bois ainsi traités seraient classés peu durables après trois ans de mise en contact avec le sol, alors que le traitement est prévu comme très durable.

Mots-clés : dépollution, bioremédiation, bois traités, cuivre chrome arsenic (CCA), champignons lignivores, pentachlorophénol (PCP), perte de masse, sol tropical.

\section{ABSTRACT}

\section{RESUMEN}

\section{CAPACITY OF TROPICAL FOREST SOILS OF FRENCH GUIANA AND RÉUNION FOR DEPOLLUTING THE WOODS IMPREGNATED WITH BIOCIDES}

Wood material for a long time was treated with fungicides or insecticides whose impact on the soil after leaching constitutes a real environmental problem. Nowadays, most of the studies on degradation of these toxic products was carried out with microorganisms which have been isolated in the laboratory. The present study sought to refine the knowledge vis-à-vis these microorganisms, especially wood-destroying fungi degrading pollutants in situ, from which few data are actually available. To decontaminate treated wood, the capacity of wooddestroying microorganisms from tropical forest soils of French Guiana and Reunion was evaluated to degrade toxic biocides. These are pentachlorophenol (PCP) and copper- chromium arsenic based compounds (CCA). Monitoring the degradation of samples of red pine, Pinus resinosa, shows that soils of French Guiana are more efficient than those of Reunion Island in terms of microbial activity vis-àvis these two biocides. A significant difference in loss of mass in specimens of red pine treated with CCA and PCP can range from single to double (respectively $18 \%$ and $30 \%$ ). These findings confirm that CCA is less leacher and less degradable than the PCP by microorganisms in the soil. According to the scale of mass loss in laboratory tests, the wood so treated would be classified very not durable after three years of contact with soil, while the treatment is expected to be very durable.

Keywords: depollution, bioremediation, treated timber, copper chromium arsenic (CCA) wood-destroying fungi, pentachlorophenol (PCP), loss of mass, tropical soil.

\section{CAPACIDAD DE SUELOS DE BOSQUES TROPICALES DE GUAYANA FRANCESA Y DE LA REUNIÓN PARA DESCONTAMINAR MADERAS IMPREGNADAS CON BIOCIDAS}

El material maderable ha sido desde hace mucho tiempo sometido a tratamientos con fungicidas o insecticidas cuyo impacto sobre el suelo después de la lixiviación constituye un problema medioambiental muy concreto. Hasta hoy, la mayor parte de los estudios sobre la degradación de estos productos tóxicos se llevó a cabo con microorganismos aislados en laboratorio. El objetivo de este estudio ha consistido en mejorar el conocimiento de estos microorganismos, especialmente los hongos destructores de la madera que degradan los contaminantes in situ, de lo cual se dispone de pocos datos. Para descontaminar la madera tratada, se evaluó la capacidad de los microorganismos de los suelos de los bosques tropicales de Guayana Francesa y de la Reunión para degradar biocidas tóxicos. Se trata del pentaclorofenol (PCP) y de compuestos a base de cobre cromo y arsénico (CCA). El seguimiento y control de la degradación de las muestras de pino rojo, Pinus resinosa, ha mostrado que los suelos de Guayana Francesa son más eficientes que los de la isla de Reunión, en términos de la actividad microbiana respecto a los dos biocidas. Una diferencia significativa en la pérdida de masa de especímenes de pino rojo tratada con CCA y el PCP puede variar de simple a doble (respectivamente $18 \%$ y $30 \%$ ). Estos resultados confirman que CCA es menos lixiviable y menos degradable por microorganismos del suelo que el PCP. De acuerdo con la escala de pérdida de masa según pruebas de laboratorio, la madera así tratada se clasificaría no duradera después de tres años de contacto con el suelo, mientras que se cuenta con un tratamiento muy duradero.

Palabras clave: descontaminación, bioremediación, madera tratada, cobre cromo arsénico (CCA), hongos xilófagos, pentaclorofenol (PCP), pérdida de masa, suelo tropical. 


\section{Le contexte}

\section{État de l'art}

Le temps conduit le bois, matière organique, vers une dégradation de sa substance, surtout lorsqu'il est exposé aux pathogènes tels que les champignons, les bactéries et les insectes. Pour tenter d'inhiber ce phénomène, plusieurs traitements de préservation ont été développés et appliqués grâce au développement de substances chimiques biocides. Les techniques utilisées sont, notamment, l'application ou l'imprégnation de surface, le trempage et différentes techniques d'imprégnation profondes, sous vide et sous pression, sous double vide (FOUGEROUSSE, 1979). Ce sont des procédés à base de produits chimiques contenant des métaux lourds ou des composés aromatiques qui permettent au matériau de ne pas subir les dégradations biotiques. Mais les produits chimiques utilisés sont hautement toxiques. En 1998, l'Union européenne a édicté la Directive biocide 98/8/CE et en 2012 le règlement relatif aux produits biocides, RPB 582/2012, applicable le $1^{\text {er }}$ septembre 2013. Le règlement vise à harmoniser l'évaluation scientifique et la commercialisation des produits biocides de telle sorte qu'ils ne présentent pas de risques pour la santé des hommes et des animaux et pour l'environnement. Cependant, ces produits posent des problèmes non résolus de toxicité, de rémanence et de devenir en fin de vie au sein des matériaux traités (MEDDE $\left.{ }^{1}, 2013\right)$. Par exemple, le bois, ainsi traité, ne peut ni être brûlé, à cause de la pollution atmosphérique que cela provoquerait, ni être réutilisé, en raison de sa toxicité (MDDEP2, 2011). Il est donc souvent entreposé, engendrant une pollution des sols sous-jacents provoquée par les produits toxiques lessivés. Bien que ces produits puissent être dégradés dans le sol par les micro-organismes, cette dégradation s'avère très lente. Parmi ces micro-organismes, les champignons lignivores sont connus pour dégrader les produits biocides et peuvent être utilisés comme agent de biorestauration (ANASTASI et al., 2008 ; KAMEI et al., 2010). GOLD et ALIC (1993) ont mis en évidence la dégradation d'une polychlorodibenzodioxine par le basidiomycète Phanerochaete chrysporium. Ces auteurs ont pu déterminer le mécanisme de dégradation mis en jeu par ce champignon de pourriture blanche en caractérisant les métabolites synthétisés et les produits d'oxydation générés à partir de la lignine impliquant notamment des manganèses peroxydases $(\mathrm{MnP})$. Un article de synthèse publié par CHANG, en 2008, fait le point sur la dégradation des composés, de type dioxines, par les micro-organismes et, en particulier, sur les deux possibilités de dégradation par les champignons soit par le biais du cytochrome P-450, soit par le biais des peroxydases et notamment la lignine peroxydase (LiP) et la MnP (VALLI et al., 1992). Par la suite, d'autres auteurs confirmeront l'intérêt de ces voies métaboliques dans la dégradation de certains composés chimiques : SAKAKI et MUNETSUNA (2010) et SUHARA et al. (2011) s'intéressent plus particulièrement au cytochrome P-450 tandis que MANJI et ISHIHARA (2004) focalisent leurs travaux sur les peroxydases. Ces enzymes, ainsi que les laccases, sont les enzymes qui catalysent le processus de dégradation extracellulaire de la lignine par les champignons lignivores de pourriture blanche (HIRATSUKA et al., 2005). Ainsi, en 2010, SAKAKI et MUNETSUNA publient un article sur la faisabilité de méthodes de bioremédiation mettant en jeu différents champignons lignivores de pourriture blanche. En 2003, ANDERSSON et al. montrent que la dégradation de dioxines chlorées nécessite l'utilisation de champignons de pourriture blanche ou fibreuse, connus pour être les acteurs de la dégradation de la lignine. D'autres auteurs comme POINTING (2001) mettent en évidence de nombreux autres champignons de pourriture blanche pouvant intervenir en tant qu'agents de bioremédiation tels que Phlebia Lindtneri, Ceriporia, Phanerochaete sordida, Bjerkandera sp., Cordyceps sinensis, Coriolus hirsutus, Phlebia radiata, Phlebia brevispora, Pseudallescheria boydii, Coprinellus spp. Par contre, la dégradation de certains métaux lourds semble incomber aux champignons de pourriture brune tels que l'espèce Antrodia vaillantii (SIERRA-ALVAREZ, 2009). En outre, même si ces champignons ne dégradent pas complètement les bois traités ou ne remédient pas totalement à la toxicité des sols pollués, ils peuvent être utilisés comme prétraitement dans le but de diminuer la quantité de substance organique avant un traitement plus radical, tel que l'incinération (VALENTIN et al., 2009).

\section{Objectif de l’étude}

En 2013, une première étude a été lancée sur l'exploration de la biodiversité microbienne au contact de bois et de sols traités au pentachlorophénol (PCP) et avec des composés à base de cuivre chrome arsenic (CCA) (ZAREMSKI et al., 2012). Les premières analyses culturales et moléculaires ont permis d'isoler et d'identifier quarante souches d'ascomycètes capables de se développer sur le bois et de résister à des polluants. En outre, l'étude indique que les produits biocides lessivés dans le sol ont un impact sur les communautés fongiques qui sont différentes selon le temps d'échantillonnage. En tant que suite logique, l'objectif de la présente étude a porté, d'une part, sur la capacité ou l'aptitude des micro-organismes des sols de Guyane française à dégrader le pin rouge, Pinus resinosa, et certains bois tropicaux et, d'autre part, sur leur faculté à dégrader ces bois, traités au PCP ou au CCA. Quant à la dégradation du bois, une comparaison entre des sols de Guyane et de la Réunion a été assurée grâce à la perte de masse, pour mettre en évidence l'activité fongique. L'ensemble de l'étude a été mené à partir de poteaux en pin rouge traités au PCP ou avec du CCA. Ce dernier est surtout utilisé pour traiter des bois exposés à l'extérieur. Le cuivre y est utilisé en tant que fongicide, l'arsenic comme insecticide et le chrome sert à fixer ces molécules dans le bois. Alors que le cuivre est assez peu toxique pour les mammifères, l'arsenic est connu pour être cancérogène pour l'homme (SIERRA-ALVAREZ, 2009). Le $\mathrm{PCP}$, en revanche, n'est plus autorisé dans les constructions en contact avec les hommes, depuis les années 1990, dans de nombreux pays. Même si son utilisation s'avère décrois-

\footnotetext{
${ }^{1}$ Ministère de l'Écologie, du Développement durable et de l'Énergie de France.

${ }^{2}$ Ministère du Développement durable, de l’Environnement et des Parcs du Québec.
} 
Un dispositif identique à celui de la Réunion a été installé dans le sol de Guyane, proche du Laboratoire des sciences du bois à Kourou. Les éprouvettes qui ont servi à la comparaison avec le sol de la Réunion ont été prélevées au bout de cinq mois de mise en terre.

\section{Mesure de la perte de masse}

Les éprouvettes prélevées pour le calcul de la perte de masse ont été identifiées, brossées, lavées et essuyées ; puis pesées une première fois aux taux d'humidité immédiatement après le lavage avec la balance Sartorius CP224S et le logiciel Software Wedge. Ensuite, les éprouvettes ont été placées dans une étuve à $103^{\circ} \mathrm{C}$ pendant 48 heures. Enfin, les éprouvettes sorties de l'étuve ont été pesées une seconde fois pour obtenir leur masse anhydre. Le calcul de la perte de masse est le suivant :

$\Delta \mathrm{M}=\left(\left(\mathrm{M}_{0}-\mathrm{M}_{1}\right) / \mathrm{M}_{0}\right) \times 100$

où :

$\Delta \mathrm{M}$ est la perte de masse en pourcentage ;

$M_{0}$ est la masse anhydre des éprouvettes en grammes avant leur mise en place dans les essais ;

$M_{1}$ est la masse anhydre des éprouvettes prélevées des dispositifs.

\section{Interprétation statistique}

L'analyse des pertes de masse des éprouvettes cherche à évaluer de possibles différences significatives de perte de masses entre plusieurs sites ou traitements. II s'est avéré que les variances de ces données n'ont pas présenté une répartition normale, même après transformation logarith-
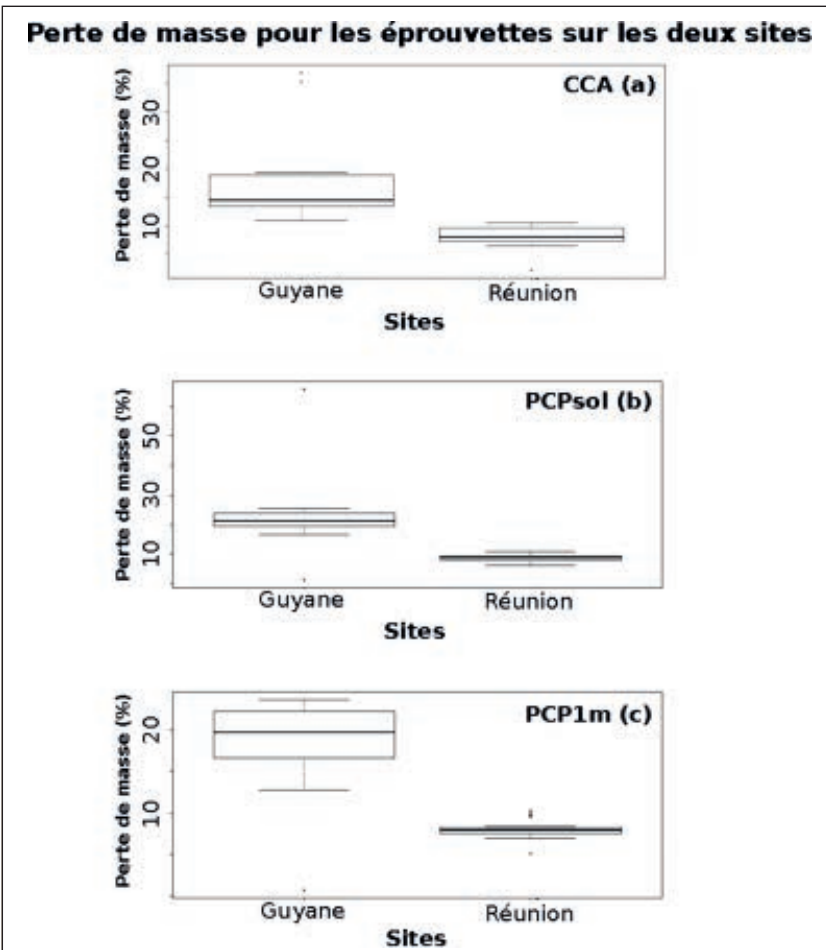

Figure 1.

Comparaison des pertes de masse d'éprouvettes de pin rouge soumises à différents traitements, CCA (a), $P C P_{\text {sol }}(b)$ et $P C P_{1 \mathrm{~m}}(\mathrm{c})$, entre deux sites de deux régions géographiques différentes (Guyane et Réunion). mique des variables, qui était la transformation la plus adaptée à la distribution, et après celle, plus générale, du Box-Cox. C'est pourquoi le type de test choisi a été une Anova non paramétrique à un critère de classification de Kruskal-Wallis. Puis, au cas où ce test permette de rejeter l'hypothèse $\mathrm{H}_{0}$ d'unicité de la population par une P-value > 0,05 , les données sont à soumettre au test de comparaison multiple de Siegel et Castellan évaluant les différences entre groupes; les analyses statistiques étant réalisées grâce au logiciel R 3.0.1.

\section{Résultats}

\section{Dégradation d'éprouvettes de pin rouge dans un sol de la Réunion}

Les différences dans la dégradation des éprouvettes de pin rouge dans un sol de Guyane et dans un sol de la Réunion ont été estimées grâce aux différences de perte de masse des échantillons dans chaque sol. Cette variation (figure 1) montre les degrés de dispersion des pertes de masse pour chaque type de sol. La perte de masse des éprouvettes mises en place à la Réunion est plus faible que la perte de celles mises en place en Guyane. Pour le CCA, cette différence est d'environ $5 \%$, et deux fois plus importante pour le PCP de l'ordre de $10 \%$.

\section{Dégradation d'éprouvettes de pin rouge dans un sol de Guyane}

\section{Perte de masse des éprouvettes traitées}

Les différences dans la dégradation des éprouvettes de pin rouge pour les trois types de traitements donnés (CCA, $P C P_{\text {sol }}, P C P_{1 \mathrm{~m}}$ ) ont été estimées grâce aux différences de perte de masse des échantillons pour chaque traitement. Cette variation (figure 2) montre les différents degrés de dispersion des pertes de masse pour chaque traitement. La perte de masse des éprouvettes traitées au CCA est légèrement dispersée autour de $18 \%$, alors que pour celles traitées au PCP elle est presque deux fois plus importante avec $30 \%$. Ainsi, les différences de perte de masse sont significatives entre les éprouvettes traitées au CCA et celles traitées au PCP. En revanche, entre les deux traitements $\mathrm{PCP}_{\text {sol }}$ et $P C P_{1 \mathrm{~m}}$, la différence n'est pas significative.

\section{Perte de masse des éprouvettes témoins}

Les conteneurs dont les éprouvettes étaient traitées au CCA et au $\mathrm{PCP}_{\text {sol }}$ contenaient également des éprouvettes témoins en pin rouge et en yayamadou. Pour ceux contenant celles traitées au $\mathrm{PCP}_{1 \mathrm{~m}}$, ils ne contenaient que des éprouvettes témoins en yayamadou. La comparaison des pertes de masse (figure 3) montre que, dans les conteneurs des traitements CCA et PCP $_{\text {sol }}$, les éprouvettes contenant le yayamadou sont totalement dégradées alors que toutes ne le sont pas dans les conteneurs $P C P_{1 \mathrm{~m}}$. Ces éprouvettes témoins ont une perte de masse moyenne de $88 \%$ (écart-type $=2,829$; variance $=5,337$ ). Les éprouvettes témoins en pin rouge ne sont jamais totalement dégradées. En fait, celles des conteneurs CCA ont une perte de masse qui varie entre $65 \%$ et $85 \%$ alors que celles des conteneurs $\mathrm{PCP}_{\text {sol }}$ varient entre $45 \%$ et $65 \%$. 
Etude de la perte de masse pour les différents traitements

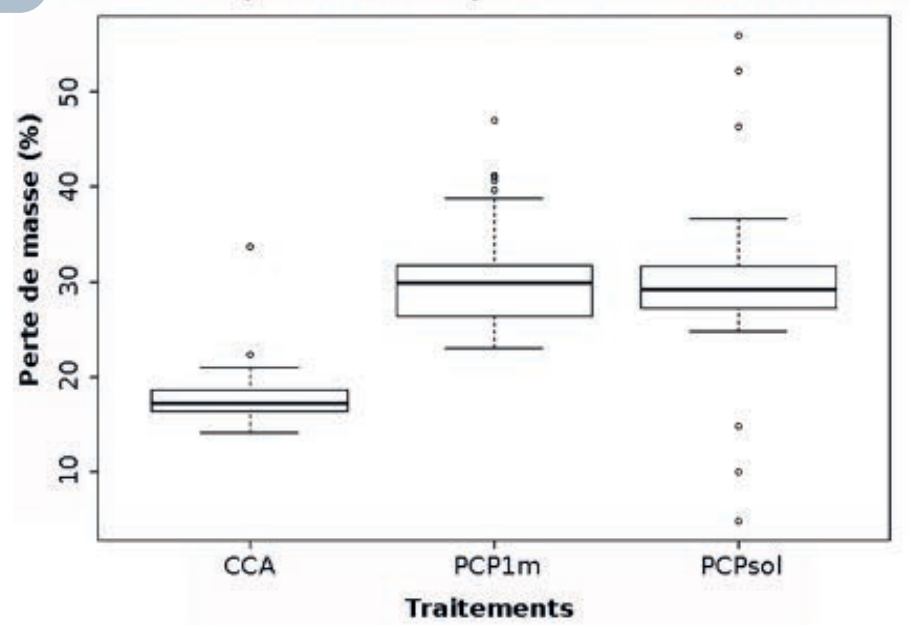

Figure 2.

Comparaison de la perte de masse pour les éprouvettes de pin rouge soumises à différents traitements.

\section{Etude de la perte de masse des éprouvettes témoins}

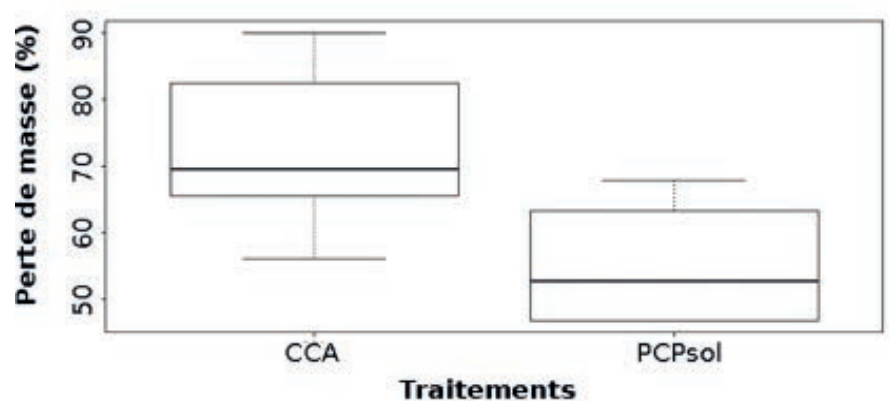

Figure 3.

Comparaison des pertes de masse des éprouvettes témoins de pin rouge pour les traitements CCA et $\mathrm{PCP}_{\text {sol }}$.

\section{Discussion}

Les essais mis en place dans un sol de Guyane et un sol de la Réunion montrent une différence significative de la dégradation des éprouvettes pour le pin rouge avec une meilleure capacité de dégradation en faveur du sol de Guyane. Le temps de maintien au sol renforce d'autant ces résultats puisque les éprouvettes mises en place à la Réunion (10 mois) ont été au sol deux fois plus longtemps que celles mises en place en Guyane (5 mois). Ces résultats soulignent l'intérêt d'établir des essais sur la dégradation des bois traités au CCA et au PCP en Guyane (Point de Combi), plutôt que sur le site de Bassin-Plat à la Réunion.

Les essais sur les bois traités montrent une différence significative de la perte de masse des éprouvettes de pin rouge traitées au CCA et au PCP, allant presque du simple au double (respectivement $18 \%$ et $30 \%$ ). D'après le barème des pertes de masse en essais laboratoire (tableau I), ces pourcentages de perte de masse, de $18 \%$ et $30 \%$, classe- raient respectivement les éprouvettes de bois traité au CCA ou au PCP comme peu durables et périssables, après trois ans de mise en contact avec le sol alors qu'elles sont traitées à l'origine pour être très durables. Cette différenciation entre les bois traités au CCA et au PCP s'observe également lors de l'analyse des communautés fongiques qui dégradent le bois. Il apparaît que le traitement CCA inhibe fortement les communautés fongiques en début d'expérimentation. À l'inverse, même si les tests statistiques ne permettent pas de conclure à une différence significative entre les traitements $C C A$ et $\mathrm{PCP}_{\text {sol }}$, la perte de masse des éprouvettes témoins dans les bacs contenant des éprouvettes traitées au CCA est plus importante que celle des éprouvettes témoins des bacs contenant des éprouvettes traitées au $\mathrm{PCP}_{\text {sol }}$. Il est à noter que la $\mathrm{P}$-value est assez faible, à peine supérieure à 0,05 ( $P$-value $=0,0550$ pour $\mathrm{df}=1$ et $2=3,681$ ) ; elle permet toutefois de signifier cette relation, qui met en évidence la plus grande lessivabilité du PCP par rapport au CCA. De plus, les éprouvettes témoins en yayamadou ont été totalement dégradées pour ces deux traitements, alors qu'elles ne le sont pas entièrement dans les conteneurs du traitement $\mathrm{PCP}_{1 \mathrm{~m}}$.

La capacité de lessivage des deux traitements est différente : le CCA est peu lessivable alors que le PCP l'est beaucoup plus (FOUGEROUSSE, 1979). Les organismes lignivores ont plus de difficultés à dégrader les éprouvettes traitées au CCA, encore très présents dans le bois, alors qu'ils ont plus de facilité à dégrader le bois traité au PCP, qui a été plus facilement lessivé. Par ailleurs, le CCA étant, de ce fait, peu répandu dans le sol des conteneurs, les micro-organismes y sont assez développés pour dégrader considérablement les éprouvettes témoins. En revanche, le PCP étant plus répandu dans le sol des conteneurs, les micro-organismes ont plus de mal à se maintenir et dégradent moins les éprouvettes témoins. Une analyse des communautés fongiques du sol au contact des éprouvettes (données non montrées) en février 2011 montre que les trois types de traitements peuvent être distingués. Cette différence se remarque également entre les deux traitements PCP car les éprouvettes en yayamadou des conteneurs $\mathrm{PCP}_{1 \mathrm{~m}}$ ne sont pas totalement dégradées alors que le yayamadou est une espèce très peu durable. Il y avait probablement davantage de PCP dans les éprouvettes traitées au $P C P_{1 \mathrm{~m}}$ que dans les éprouvettes traitées au $\mathrm{PCP}_{\text {sol }}$. En effet, ce dernier traitement correspond au bas des poteaux électriques en contact avec le sol, où le lessivage et l'activité biologique étaient beaucoup plus importants quand ils étaient mis en œuvre en tant que poteau. En faisant le parallèle entre ces résultats et ceux de ANDERSSON et al. (2003), ayant trait à la dégradation de certaines toxines par les micro-organismes, une question peut se poser : cette diminution de l'activité de dégradation par les micro-organismes lignivores serait-elle due aux produits secondaires de dégradation du PCP ? Il serait judicieux de les identifier pour en estimer leur toxicité. Les différences entre les conteneurs pour un même traitement confirment le résultat d'une différence de perte de masse entre les éprouvettes traitées au CCA et celles traitées au PCP et d'une unicité dans les pertes de masse pour les traitements $P C P_{\text {sol }}$ et $P C P_{1 \mathrm{~m}}$. 
Tableau I.

Barème des pertes de masse en essais laboratoire exprimées en pourcentage.

\begin{tabular}{|l|l|} 
Perte de masse en laboratoire & Classe de durabilité \\
\hline Supérieure à $25 \%$ & Périssable \\
\hline Entre 15 et $25 \%$ & Peu durable \\
\hline Entre 10 et $15 \%$ & Moyennement durable \\
\hline Entre 5 et $10 \%$ & Durable \\
\hline Inférieure ou égale à $5 \%$ & Très durable \\
\hline
\end{tabular}

\section{Conclusion et perspectives}

Le bois est un matériau qui, selon l'essence utilisée, peut être très sensible à son environnement. Il convient donc de renforcer sa durabilité naturelle par des procédés chimiques ou naturels. Jusque dans les années 1980, une première génération d'agents biocides a été utilisée, notamment le pentachlorophénol (PCP) formulé dans des solvants pétroliers. Le $P C P$ est un dérivé goudronné facilement lessivé par l'eau, qui par son ruissellement pollue le sol dans lequel elle s'infiltre. Ce produit a donc été interdit par les autorités et remplacé par un biocide de deuxième génération : les composés à base de cuivre chrome arsenic (CCA), légèrement moins toxiques et plus efficaces, mais interdits aujourd'hui par la Directive européenne dite «biocide ». Actuellement, des produits biocides de troisième génération sont commercialisés, comme le couple propiconazole/tébuconazole ou d'autres combinaisons différentes de fongicides en fonction des usages.

La question du recyclage ou de la destruction des bois traités avec les produits de première et deuxième génération se pose aujourd'hui avec une particulière acuité du fait de l'évolution de la réglementation qui contraint les industriels du bois à gérer ces produits arrivés en fin d'utilisation. Ce problème concerne non seulement les bois en fin de vie retirés chaque année, mais aussi les stocks qui se sont accumulés depuis des années, souvent dans des conditions non adaptées, et les impacts sur l'environnement sont importants : pollution de l'air, du sol, des nappes phréatiques...

Les micro-organismes des sols de Guyane présentent un avantage majeur : à l'inverse des techniques chimiques ou thermiques, ils dégradent les polluants au lieu de simplement les extraire. En outre, contrairement aux bactéries qui sont spécifiques d'un polluant, ces micro-organismes, tels que les champignons lignivores, sont capables de dégrader un panel étendu de substances toxiques à l'aide de nombreuses enzymes extracellulaires qu'ils sécrètent comme la lignine peroxydase, la manganèse peroxydase et le cytochrome P-450.
Deux questions restent toutefois en suspens concernant le PCP : la diminution de l'activité microbienne, mise en évidence par la perte de masse des éprouvettes témoins, estelle seulement due au lessivage du PCP ? Les communautés microbiennes pourront-elles dégrader le PCP lessivé ? Afin de répondre à ces questions, la mise en place d'autres essais a été programmée sur sol du site de Kourou dans le cadre de prochaines études. Il s'agira d'identifier ces microorganismes et de les caractériser par des méthodes culturales et moléculaires. D’autres études moléculaires et biochimiques seront menées sur l'implication de ces enzymes dans la dégradation de composés chimiques très toxiques à la fois pour l'homme et l'environnement. Une autre perspective sera la valorisation des bois qui, grâce à leur dépollution, peuvent être recyclés et alimenter des filières consommatrices de bois de rebut; à titre d'exemple, pour la production d'énergie à partir de biomasse. D'autres voies de valorisation sont aussi à étudier pour des finalités écologiques, environnementales, telles que le traitement de boues d'épuration, la dépollution des sites de stockage ou les accélérateurs de compostage à base de bois.

\section{Remerciements}

Les auteurs remercient l'Institut de recherche d'HydroQuébec pour le financement du projet, le personnel du Laboratoire des sciences du bois du Cirad à Kourou en Guyane française, ainsi que les agents du Cirad de la station de Bassin-Plat à Saint-Pierre de La Réunion.

\section{Références bibliographiques}

ALTSCHUL S. F., GISH W., MILLER W., MYERS E. W., LIPMAN D. J., 1990. Basic local alignment search tool. Journal of Molecular Biology, 215: 403-410.

ANDERSSON B. E., LUNDSTEDT S., TORNBERG K., SCHNÜRER Y., ÖBERG L. G., MATTIASSON B., 2003. Incomplete degradation of polycyclic aromatic hydrocarbons in soil inoculated with wood rotting fungi and their effect on the indigenous soil bacteria. Environmental Toxicology and Chemistry, 22: 1238-1243.

ANASTASI A., VARESE G. C., BOSCO F., CHIMIRRI F., FILIPELLO MARCHISIO V., 2008. Bioremediation potential of basidiomycetes isolated from compost. Bioresource Technology, 99: 6626-6630.

BERRIN J. G., NAVARRO D., COUTURIER M., OLIVE C., GRISEL S., HAON M., TAUSSAC S., LECHAT C., COURTECUISSE R., FAVEL A., COUTINHO P. M., LESAGE-MEESSEN M., 2012. Exploring the natural fungal biodiversity of tropical and temperate forests toward improvement of biomass conversion. Applied and Environmental Microbiology, 78 (18): 6483-6490.

BLANCANEAUX P., 1981. Essai sur le milieu naturel de la Guyane française. Paris, France, Orstom, Travaux et documents de l'Orstom 137, 126 p. 
CHANG Y.-S., 2008. Recent developments in microbial biotransformation and biodegradation of dioxins. Journal of Molecular Microbiology and Biotechnology, 15: 152-171.

FOUGEROUSSE M., 1979. Préservation des menuiseries contre la pourriture. Bois et Forêts des Tropiques, 183 : 49-66.

GOLD M. H., ALIC M., 1993. Molecular biology of the lignindegrading basidiomycete Phanerochaete chrysosporium. Microbiological Reviews, 57 (3): 605-622.

HIRATSUKA N., OYADOMARI M., SHINOHARA H., TANAKA H., WARIISHI H., 2005. Metabolic mechanisms involved in hydroxylation reactions of diphenyl compounds by the lignin-degrading basidiomycete Phanerochaete chrysosporium. Biochemical Engineering Journal, 23: 241-246.

KAMEI I., TAKAGI K., KONDO R., 2010. Bioconversion of dieldrin by wood-rotting fungi and metabolite detection. Pest Management Science, 66: 888-891.

MANJI S., ISHIHARA A., 2004. Screening of tetrachlorodibenzo-p-dioxin-degrading fungi capable of producing extracellular peroxidases under various conditions. Applied Microbiology and Biotechnology, 63: 438-444.

MDDEP (Ministère du Développement Durable, de l'Environnement et des Parcs), 2011. Lignes directrices relatives à la gestion du bois traité. Québec, Ministère du Développement durable, de l'Environnement et des Parcs, Direction des matières résiduelles et des lieux contaminés, 28 p.

MEDDE (Ministère de l'Écologie, du Développement durable et de l'Énergie), 2013. France. [en ligne, 29/05/2013], www.developpement-durable.gouv.fr.

MILESKI G. J., BUMPUS J. A., JUREK M. A., AUST S. D., 1988. Biodegradation of pentachlorophenol by the white rot fungus Phanerochaete chrysosporium. Applied and Environmental Microbiology, 54 (12): 2885-2889.

MULLIS K. B., FALOONA F. A., 1985. Specific synthesis of DNA in vitro via a polymerase-catalyzed chain reaction. Methods in Enzymology, 155: 335-350.

POINTING S. B., 2001. Feasibility of bioremediation by white-rot fungi. Applied Microbiology and Biotechnology, 57 (1-2): 20-33.

SAKAKI T., MUNETSUNA E., 2010. Enzyme systems for biodegradation of polychlorinated dibenzo-p-dioxins. Applied Microbiology and Biotechnology, 88 (1): 23-30.

SATO A., WATANABE T., WATANABE Y., KURANE R., 2003. Enhancement of biodegradation of 2,7-dichlorodibenzo-pdioxin by addition of fungal culture filtrate. World Journal of Microbiology and Biotechnology, 18: 439-441.

SIERRA-ALVAREZ R., 2009. Removal of copper, chromium and arsenic from preservative-treated wood by chemical extraction-fungal bioleaching. Waste Management, 29: 1885-1891.

SUHARA H., KAMEI I., MAEKAWA N., KONDO R., 2011. Biotransformation of polychlorinated dibenzo-p-dioxin by Coprinellus species. Mycoscience, 52: 48-52.
VALENTÍN L., KLUCZEK-TURPEINEN B., OIVANEN P., HATAKKA A., STEFFEN K., TUOMELA M., 2009. Evaluation of basidiomycetous fungi for pretreatment of contaminated soil. Journal of Chemical Technology and Biotechnology, 84: 851-858.

VALLI K., WARIISHI H., GOLD M. H., 1992. Degradation of 2,7-dichlorodibenzo-p-dioxin by the lignin-degrading basidiomycete Phanerochaete chrysosporium. Journal of Bacteriology, 174 (7): 2131-2137.

WHITE T. J., BRUNS T. D., LEE S. B., TAYLOR J. W., 1990. Amplification and direct sequencing of fungal ribosomal RNA genes for phylogenetics. In: Innis M. A., Gelfand D. H., Sninsky J. J., White T. J. (Eds). PCR Protocols: A Guide to Methods and Applications. San Diego, CA, États-Unis, Academic Press, 315-322.

WINQUIST E., VALENTIN L., MOILANEN U., LEISOLA M., HATAKKA A., TUOMELA M., STEFFEN K. T., 2008. Development of a fungal pre-treatment process for reduction of organic matter in contaminated soil. Journal of Chemical Technology and Biotechnology, 84: 845-850.

ZAREMSKI A., PALANTI S., MANNUCCI M., GASTONGUAY L., LE FLOCH G., 2011. Molecular diagnosis by PCR-DHPLC technique of wood-decay fungi in historical buildings in Italy. Proligno, 7 (4): 92-97.

ZAREMSKI A., GASTONGUAY L., ZAREMSKI C., CHAFFANEL F., BEAUCHÊNE J., LE FLOCH G., 2012. Biodegration of treated wood waste by native fungal communities of tropical soil in French Guiana. In: Proceedings IRG Annual Meeting (IRG/WP 12-50285), Kuala Lumpur, Malaysia, 2012/05/06-10. International Research Group on Wood Protection Annual Meeting 43. 